\title{
Short-term antidepressant treatment has long-lasting effects, and reverses stress- induced decreases in bone features in rats
}

\author{
S. H. Lee ${ }^{1,10}$, C. A. Mastronardi, ${ }^{1,2}$, R. W. Li ${ }^{3}$, G. Paz-Filho ${ }^{1,11}$, E. G. Dutcher ${ }^{4,5}$, M. D. Lewis ${ }^{4,6}$, A. D. Vincent ${ }^{7}$, P. N. Smith ${ }^{3,8}$,
} S. R. Bornstein ${ }^{9}$, J. Licinio ${ }^{4,6,12}$ and M. L. Wong ${ }^{4,6,13}$

\begin{abstract}
Antidepressants are among the most-prescribed class of drugs in the world and though weight gain is a common outcome of antidepressant treatment, that effect is not well understood. We employed an animal model comprised of 2 weeks of chronic restraint stress with antidepressant treatment, followed by diet-induced obesity. We showed that short-term antidepressant treatment had long-lasting effects, not only leading to weight gain, but also enhancing trabecular and cortical bone features in rats; therefore, weight gain in this model was different from that of the classic diet-induced obesity. Late in the post-restraint recovery period, antidepressant-treated animals were significantly heavier and had better bone features than saline-treated controls, when assessed in the distal femoral metaphysis. The propensity to gain weight might have influenced the rate of catch-up growth and bone allometry, as heavier animals treated with fluoxetine also had enhanced bone features when compared to non-stressed animals. Therefore, shortterm antidepressant treatment ameliorated the long-term effects of stress on body growth and bone. Growth and bone structural features were associated with leptin levels, and the interaction between leptin levels and antidepressant was significant for bone mineral content, suggesting that short-term antidepressants in the context of long-term diet-induced obesity modified the role of leptin in bone formation. To our knowledge this is the first study reporting that short-term antidepressant treatment has long-lasting effects in restoring the effects of chronic stress in body weight and bone formation. Our findings may be relevant to the understanding and treatment of osteoporosis, a condition of increasing prevalence due to the aging population.
\end{abstract}

\section{Introduction}

Major depressive disorder (MDD) and obesity are both common heterogeneous disorders of complex etiology, and pronounced public health impact ${ }^{1,2}$. According to the data from the World Health Organization (WHO), MDD has become the second most prevalent cause of illnessinduced disability, affecting 350 million people

\footnotetext{
Correspondence: J. Licinio (LicinioJ@upstate.edu) or M. L. Wong (wongma@upstate.edu)

'John Curtin School of Medical Research, College of Health and Medicine, Australian National University, Canberra, ACT 0200, Australia ${ }^{2}$ Neuroscience Group (NeUROS), Institute of Translational Medicine, School of Medicine and Health Sciences, Universidad del Rosario, Bogotá, Colombia Full list of author information is available at the end of the article.

Share senior authorship: J. Licinio, M. L. Wong
}

worldwide ${ }^{3}$. Concomitantly, obesity is a debilitating epidemic, affecting $36.5 \%$ of US adults ${ }^{4}$. Currently, given the high prevalence of obesity and mood disorders, it is conceivable that nearly $25 \%$ of the cases of obesity may be attributable to the association with $\mathrm{MDD}^{5}$. Crosssectional and longitudinal studies have been conducted in order to understand the casual relationship between MDD and obesity ${ }^{6-8}$. Both disorders have in common the dysregulation of the hypothalamic-pituitary-adrenal axis, which is persistently activated during chronic stress ${ }^{9}$.

In the USA, antidepressant drugs were the second most prescribed class of drugs in 2011-2014 ${ }^{4}$. Weight gain is a common outcome of antidepressant treatment. The interplay between MDD, obesity, and antidepressant-

\section{(c) The Author(s) 2019}

(c) (i) Open Access This article is licensed under a Creative Commons Attribution 4.0 International License, which permits use, sharing, adaptation, distribution and reproduction c. in any medium or format, as long as you give appropriate credit to the original author(s) and the source, provide a link to the Creative Commons license, and indicate if changes were made. The images or other third party material in this article are included in the article's Creative Commons license, unless indicated otherwise in a credit line to the material. If material is not included in the article's Creative Commons license and your intended use is not permitted by statutory regulation or exceeds the permitted use, you will need to obtain permission directly from the copyright holder. To view a copy of this license, visit http://creativecommons.org/licenses/by/4.0/. 
induced weight gain is complex. Though acute selective serotonin reuptake inhibitor (SSRI) treatment leads to weight loss, chronic SSRI treatment may lead to weight gain $^{10-12}$. The discontinuation rate for antidepressant treatment is high within 2 months of treatment initiation, ranging from $70 \%$ for fluvoxamine, to $45 \%$ for fluoxetine (FX) and $40 \%$ for sertraline; ${ }^{13-15}$ thus, the lifetime prevalence of antidepressant exposure is very high. Based on the model that we have described previously ${ }^{16}$, here we combined 2-weeks of recurrent restraint stress and antidepressant treatment, followed by long-term diet-induced obesity; and referred it as the stress-antidepressant and diet-induced obesity (SADIO) model.

We hypothesized that increased body weight related to antidepressant treatment in the SADIO model had different pathophysiological mechanisms from those of the diet-induced obesity model. In the SADIO model described in this paper, we show that previously described body changes in the post-stress acclimation and recovery period $^{17}$ included increased bone length, weight and structural changes. Furthermore, there was a signficant association between leptin and bone mineral content (BMC) in the SADIO model, which was not present in animals not exposed to antidepressants.

\section{Methods and materials \\ Animals}

Male Sprague-Dawley rats (170-190 g, 5-6 weeks old, Animal Resources Centre, Murdoch, WA, Australia) were housed one per cage at $24{ }^{\circ} \mathrm{C}$ and with a 12-h light/dark schedule (07:00 h to 19:00 h) in a stress-free environment. All the animal experimental procedures conducted were approved under protocol number J.MB.50.10, Animal Experimentation Ethics Committee, The Australian National University, Australia.

\section{SADIO animal paradigm}

Animals were randomly allocated into four groups: three received chronic restraint-stress (CRS) and one group did not receive CRS (NR group, $N=30$ ). The NR group did not receive CRS or intraperitoneally (i.p.) injections, but received the same dietary schedule as the CRS groups. For overview of the experimental protocol see supplementary Figure S1. Prior to CRS, there was no significant difference in body weight between the groups. CRS was applied from experimental days 5 to 19 for $6 \mathrm{~h}$ (from 9:00 to 15:00 h) using flat-bottom clear acrylic restrainers (Cat no. 544-RR Plas Labs, Lansing, MI, USA). During the CRS period, CRS groups received daily treatment with imipramine [IM, $N=13,10 \mathrm{mg} / \mathrm{kg}$ i.p.; Sigma-Aldrich, St Louis, MO, USA], fluoxetine (FX, $N=14,10 \mathrm{mg} / \mathrm{kg}$ ip; Eli Lilly, Indianapolis, Indiana, USA) or saline solution (CS, $N=10,0.9 \%$ sodium chloride solution ip, Phebra, Lane Cove West, NSW,
Australia) immediately prior to the CRS procedure. Imipramine was prepared in $24.5 \mathrm{mg} / \mathrm{mL}$ in $0.9 \%$ sodium chloride solution by mild vortexing and $\mathrm{pH}$ was adjusted to 7.4. The solution was further diluted and the final concentration of the imipramine was $4.9 \mathrm{mg} / \mathrm{mL}$ in $0.9 \%$ sodium chloride solution. Fluoxetine hydrochloride was prepared $5.6 \mathrm{mg} / \mathrm{mL}$ in $0.9 \%$ sodium chloride due to its low solubility and $\mathrm{pH}$ was adjusted to 7.4. The solution was further diluted to final concentration of $4.9 \mathrm{mg} / \mathrm{mL}$ in $0.9 \%$ sodium chloride solution. Solutions were filtered with a $0.22-\mu \mathrm{m}$ filter (EMD Millipore ${ }^{\mathrm{Tx}}$ SLGP033RS, Ontario, Canada). After the CRS period, all groups of animals received a high-fat diet (18.6\% fat semi-pure rodent diet SF10-020, Specialty Feeds, Glen Forrest, WA, Australia) to induce obesity from day 19 to 296 (276 days). Researcher could not be blinded to the experimental groups as daily i.p. injection of specific treatment group was necessary from days 5 to 19. We did not conduct power analysis prior to this study as there was no study using our animal model for 2 weeks of CRS followed by induced obesity from day 19 to 296. Body weight and food intake were measured daily during the CRS period and twice weekly thereafter. Due to the fact that rodents, such as Sprague-Dawley rats, display variable weight gain when fed a high-fat diet, animals were classified into subgroups of animals that gain significant weight (obesity prone) or not (obesity resistant $)^{18}$. Therefore, we conducted analyses to understand whether the ability to gain weight during diet-induced obesity had significant effects in our model. Within each group, rats in the upper $50 \%$ of body weights were classified as the obesity-prone subgroup based on their body weight at the end of the experiment, and animals in the lower $50 \%$ were classified as the obesity-resistant subgroup.

\section{Behavioral testing}

Open-field tests and elevated plus maze were conducted during the post-stress acclimation period (experimental weeks 3-12), equipped with a camera tracking system (Viewer II system, Biobserve GmbH, Bonn, Germany). Open-field test: Trials were conducted for $30 \mathrm{~min} / \mathrm{sessions}$ from 11:00 to 16:00 h. Rats were placed in the center of the field $(48.8 \mathrm{~cm} \times 48.8 \mathrm{~cm} \times 50 \mathrm{~cm})$. Total distance (TD), center distance $(\mathrm{CD})$, and center distance to total distance $(\mathrm{CD} / \mathrm{TD})$ ratio were obtained and used as an index of anxiety $^{16}$. Groups were CRS treated with saline, $\mathrm{N}=11$; FX, CRS treated with fluxotine, $\mathrm{N}=14$, and IM, CRS treated with imipramine, $\mathrm{N}=11$. Their means were subsequently averaged along the 10 sessions. Elevated plus maze (EPM): During the post-stress acclimation period (day 257), EPM test was performed to measure the level of anxiety-like behavior. EPM was elevated from the floor with two open arms and two closed arms $(50 \mathrm{~cm} \times 13 \mathrm{~cm})$. 
Rats were placed in the middle of the maze facing a closed arm; trials were conducted for $5 \mathrm{~min} /$ session. The number of entries into the open arms was counted and the percentage of time spent in the open arms (ratio of open arms time/closed arms time) was calculated. The groups were CS, $N=11$; FX, $N=12$; IM, $N=11$. Researchers were blinded while conducting behavioral tests.

\section{Dual energy X-ray absorptiometry (DXA) for body composition analysis and body length measurements}

Body composition analysis of subsets of obesity-prone (upper 30\%) and obesity-resistant (lower 30\%) animals were obtained under anesthesia using the GE Lunar PIXImus equipment (Madison, WI, USA) and the Lunar imaging software (ver.1.46) according to standard procedures (see supplementary methods for detailed methodology). The groups were CS, $N=4$; FX, $N=5$; IM, $N=$ 4; NR, $N=10$.

\section{Microtomography (Micro-CT)}

Micro-CT imaging of hind femurs was obtained from all animals using the Skyscan micro-CT equipment (Bruker, Kontich, Belgium). Distal femora were scanned submerged in $70 \%$ ethanol at a resolution of $21.3 \mu \mathrm{m}$. Transverse plane images were obtained from reconstructed images using the DataViewer software (Skyscan), and distal metaphyseal volume and middiaphyseal cortical geometry were generated by a binarized image program (CtAnalyzer software, Skyscan) (see supplementary methods for detailed methodology). The groups were CS, $N=10$; FX, $N=14$; and IM, $N=13$, groups. NR, $N=28$.

\section{Quantitative real time PCR (qPCR)}

cDNA samples were tested in triplicate for the following rat genes: rat $\operatorname{Igf} 1$ in liver tissue, groups were CS, $N=9$; FX, $N=12$; IM, $N=13$; NR, $N=25$; and 8 target genes in adipose tissue: Tnf, Slc2a4, Pparg, Adipoq, Fasn, Lpl, Lipe, and Ppargcla (see supplementary methods for detailed methodology and primer sequences), groups were NR, $N$ $=11$ IM, $N=11$; FX, $N=12$; and CS, $N=8$.

\section{Immunoassays}

Commercial immunoassay kits were used to determine plasma IGF-1(CS, $N=7$; FX, $N=8$; IM, $N=8$; NR, $N=$ 16), leptin (CS, $N=10$; FX, $N=14$; IM, $N=13$; NR $N=$ 30 ), triglyceride (CS, $N=10$; FX, $N=14$; IM, $N=13$; NR, $N=27$ ), total cholesterol (CS, $N=10$; FX, $N=14$; IM, $N$ $=13$; NR, $N=30$ ), free fatty acid (CS, $N=10$; FX, $N=14$; IM, $N=13$; NR, $N=30$ ), vanillylamandelic acid (CS, $N=$ 10; FX, $N=14$; IM, $N=13$; NR, $N=26$ ), and pituitary GH (CS, $N=6$; FX, $N=7$; IM, $N=6$; NR, $N=10$ ), following the manufacturers' protocols (see supplementary methods for detailed methodology).

\section{Statistical analysis}

Piecewise non-linear mixed effects growth curve models were constructed to estimate rat weights, $y$, post-stress over two periods: (A) days 20-60 and (B) day 60 onward. Analyses performed in R v3.4.1 (Vienna, Austria) using the nlme package (see supplementary methods for detailed statistical analysis $)^{19}$.

Group comparisons were done by one-way analysis of variance (ANOVA) or non-parametric test of KruskalWallis when the variances were not equal among treatment groups, and respectively followed by Tukey's or Dunn's post hoc test, using the GraphPad Prism 5.0 software (La Jolla, CA, USA) (see supplementary methods for detailed statistical analysis).

Associations between the five body composition outcomes [bone mineral content (BMC), bone mirenal density (BMD), \% fat, body length, body weight) with four biochemical measures (log-transformed plasma leptin, total cholesterol, triglyceride, and fatty acids levels) were tested using linear regressions (see supplementary methods for detailed statistical analysis).

\section{Results}

Two-week antidepressant treatment improves body weight recovery during the post-stress period

Immediately post CRS, groups treated with FX, imipramine (IM) or vehicle (CS) had significantly lower body weight $\left(\hat{\beta}_{0}^{1}\right.$ all $\left.P<0.001\right)$ than the non-stressed reference (NR) group (SI Appendix, Table S1A). During the post CRS period, the group treated with FX gained more body weight than the saline control (CS) group (Table S1 B, $P$ $=0.03$ ). Furthermore, the FX group became heavier than the NR group (Fig. 1a, b). We did not detect differences in the weights of the IM and CS-treated rats $(P=0.10)$.

\section{Chronic stressed obesity prone and obesity resistant subgroups treated with antidepressant had better weight recovery in the post-acclimation period}

The log-likelihood ratio tests indicated the presence of interactions between treatment and obesity-prone groups in both periods (both $P<0.0001$; SI Appendix, Table S1 $\mathrm{BC}$ ). In the obesity-prone (upper $50 \%$ body weight) subgroups, the FX-treated group gained more body weight than the other CRS-treated groups (obesity prone: CS vs FX $P<0.0001$, IM vs FX $P=0.01$; Fig. $1 \mathrm{c}$, and SI Appendix Table S1 B). In contrast, amongst the obesityresistant (lower $50 \%$ body weight) stressed subgroups, body weights did not differ (Fig. 1d, and SI Appendix Table S1 B).

\section{Chronic stressed FX-treated animals were less anxious}

During the post-restraint stress period (weeks 3-12), we conducted behavioral tests on animals that had undergone CRS. The FX group was significantly less anxious 


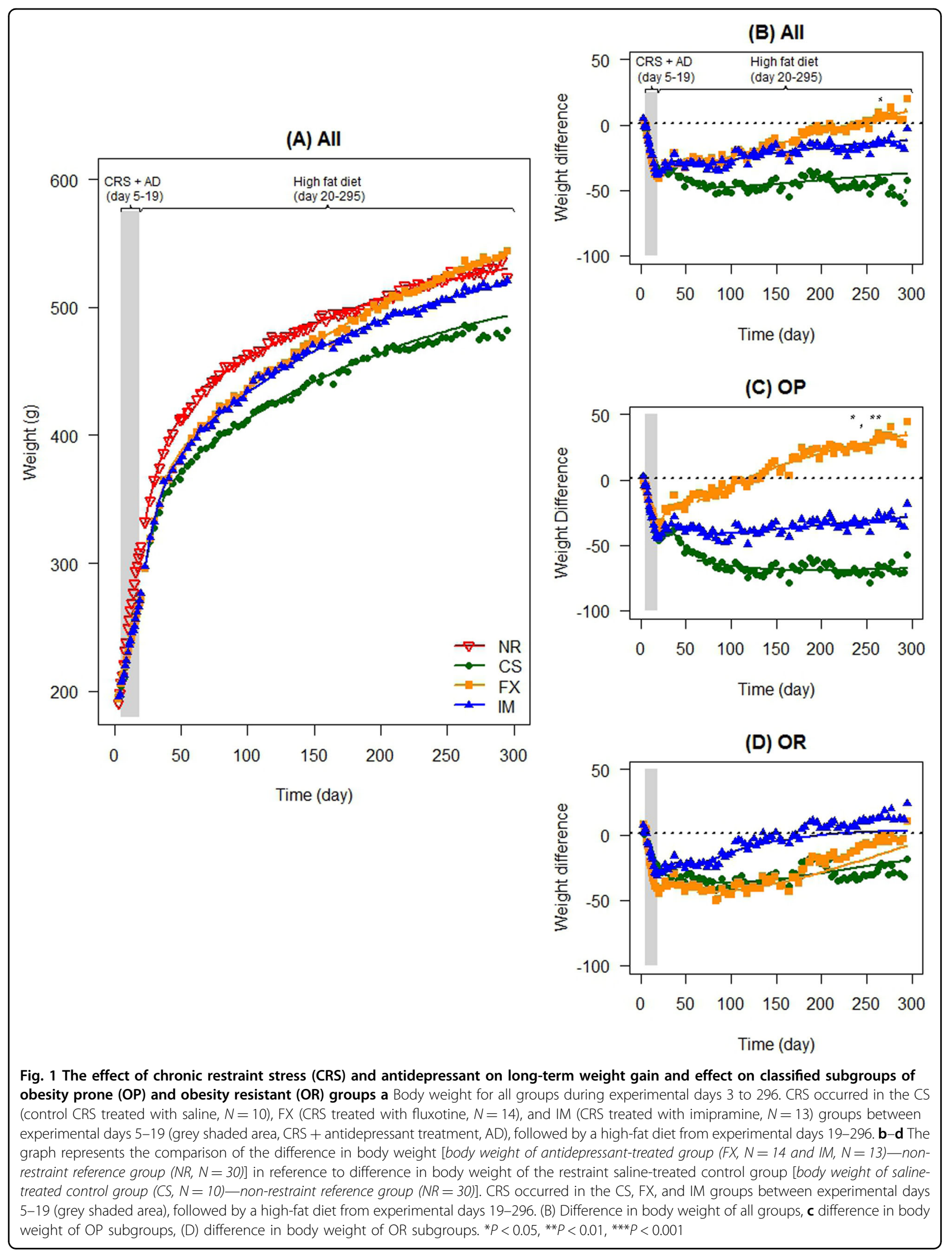



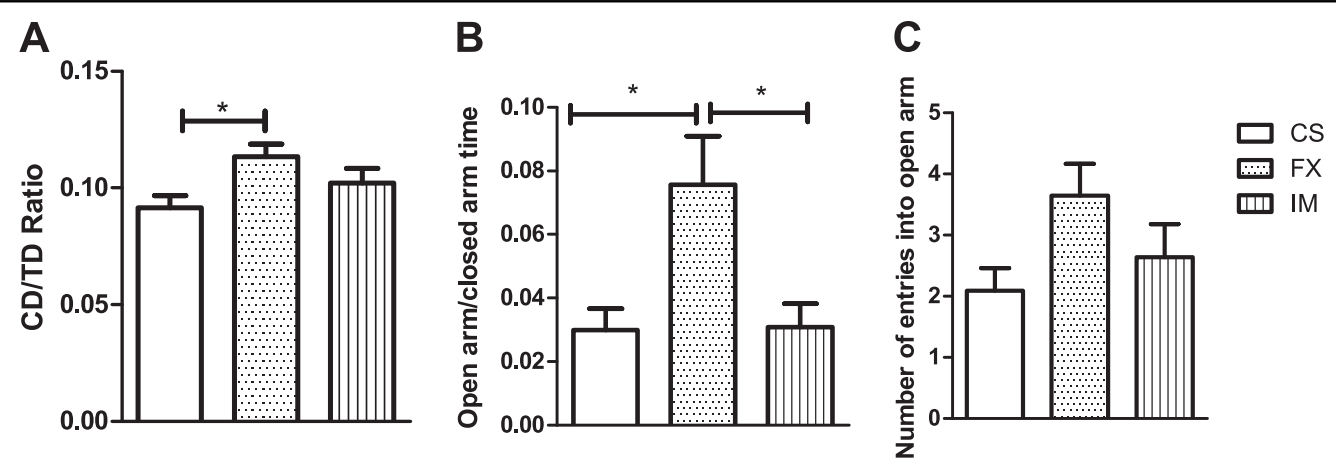

Fig. 2 Interaction between exposure to chronic restraint stress (CRS), short-term antidepressant treatment and high-fat diet, and effects on anxiety and locomotor activity during the post-restraint stress period a Locomotor activity in the open field. (CD/TD) ratio in the openfield box during 30 min of locomotor activity testing; CS, CRS treated with saline, $N=11$; FX, CRS treated with fluxotine, $N=14$, and IM, CRS treated with imipramine, $N=11$. b, $\mathbf{c}$ Elevated plus maze. $\mathbf{b}$ Time spent in open arm to closed arm ratio. $\mathbf{c}$ Number of entries into open arm. $C S, N=11 ; F X, N=$ $12 ; \mathrm{IM}, N=11$. Results are shown as means \pm s.e.m. ${ }^{*} P<0.05$

than the CS group, based on the open field test measure of $\mathrm{CD} / \mathrm{TD}$ ratio, and on the open/closed arm ratio on the elevated plus maze test (both $P<0.05$, Fig. 2a, b, and SI Appendix, Table S2).

\section{Bone morphological features were enhanced in chronic stressed antidepressant-treated animals}

Our findings support that the FX group had significantly enhanced trabecular and cortical bone microCT features (Fig. 3a). Percentage of trabecular bone volume/total volume ratio (BV/TV \%), and trabecular thickness and number were significantly greater in the FX group in comparison to the NR group (respectively, $P<$ $0.01, P<0.05$, and $P<0.05$, Fig. $3 \mathrm{a}-\mathrm{d}$, and SI Appendix, Table S3). Cortical bone features, such as mean total cross-sectional bone area and thickness, were also significantly greater in the FX group in comparison to the NR group (respectively, $P<0.01, P<0.001$, and Fig. $3 \mathrm{e}, \mathrm{f}$, and SI Appendix, Table S3). The FX group also had mean total cross-sectional thickness significantly greater than the CS groups $(P<0.05$, Fig. 3f, and SI Appendix, Table S3). The femur length was significantly greater in the IM in comparison to the CS group $(P<0.05$, Fig. $3 \mathrm{~g}$, and SI Appendix, Table S3).

\section{Hepatic Igf1 (insulin growth factor 1) mRNA levels were increased in chronic stressed animals}

At the end of the experiment, hepatic Igf1 gene expression was significantly higher in all CRS groups in comparison to the NR group $(P<0.05$ for CS and FX, and $P<0.01$ for IM, Fig. 3h, and SI Appendix, Table S2). However, IGF-1 and growth hormone (GH) plasma levels were not significantly different between the groups (respectively, Fig. 3I, j, and SI Appendix, Table S2).
Chronic stressed obesity prone saline-treated animals had lower body weight and accumulated food intake, and higher food intake ratio

Obesity-prone subgroups had significantly different body weight and accumulated higher food intake at the end of the experiment. Obesity-prone stressed animals treated with vehicle (obesity prone CS) were significantly lighter than obesity-prone FX and obesity-prone NR animals (Fig. 4a, respectively, $P<0.05$ and $P<0.01$, and SI Appendix, Table S2), and their accumulated food intake was also significantly lower (Fig. 4b, both at $P<0.05$, and SI Appendix, Table S2). Food efficiency (food intake/ $\Delta$ body weight) of the obesity-prone FX subgroup was significantly lower than the obesity-prone CS and obesityprone NR subgroups, while obesity-prone IM subgroup was significantly lower than the obesity-prone NR subgroup (Fig. 4c, $P<0.05, P<0.001$, and $P<0.05$, respectively, and SI Appendix, Table S2).

\section{Chronic stressed obesity prone saline-treated animals had lower lean and fat mass}

Obesity-prone stressed animals treated with saline (obesity-prone CS) had significantly lower lean mass and fat mass in comparison to the obesity-prone NR subgroup, and lower lean mass than obesity-prone FX animals (respectively, $P<0.05, P<0.01, P<0.001$, Fig. $4 \mathrm{~d}$, e, and SI Appendix, Table S2). The obesity-prone IM subgroup also had lower lean mass than the obesity-prone NR and the obesity-prone FX subgroups (respectively, $P<0.05$ and $P<0.001$, Fig. $4 \mathrm{~d}$, and SI Appendix, Table S2). However, there were no significant differences between the obesity-resistant subgroups in body weight, accumulated food intake, and food efficiency (SI Appendix, Table S2). 


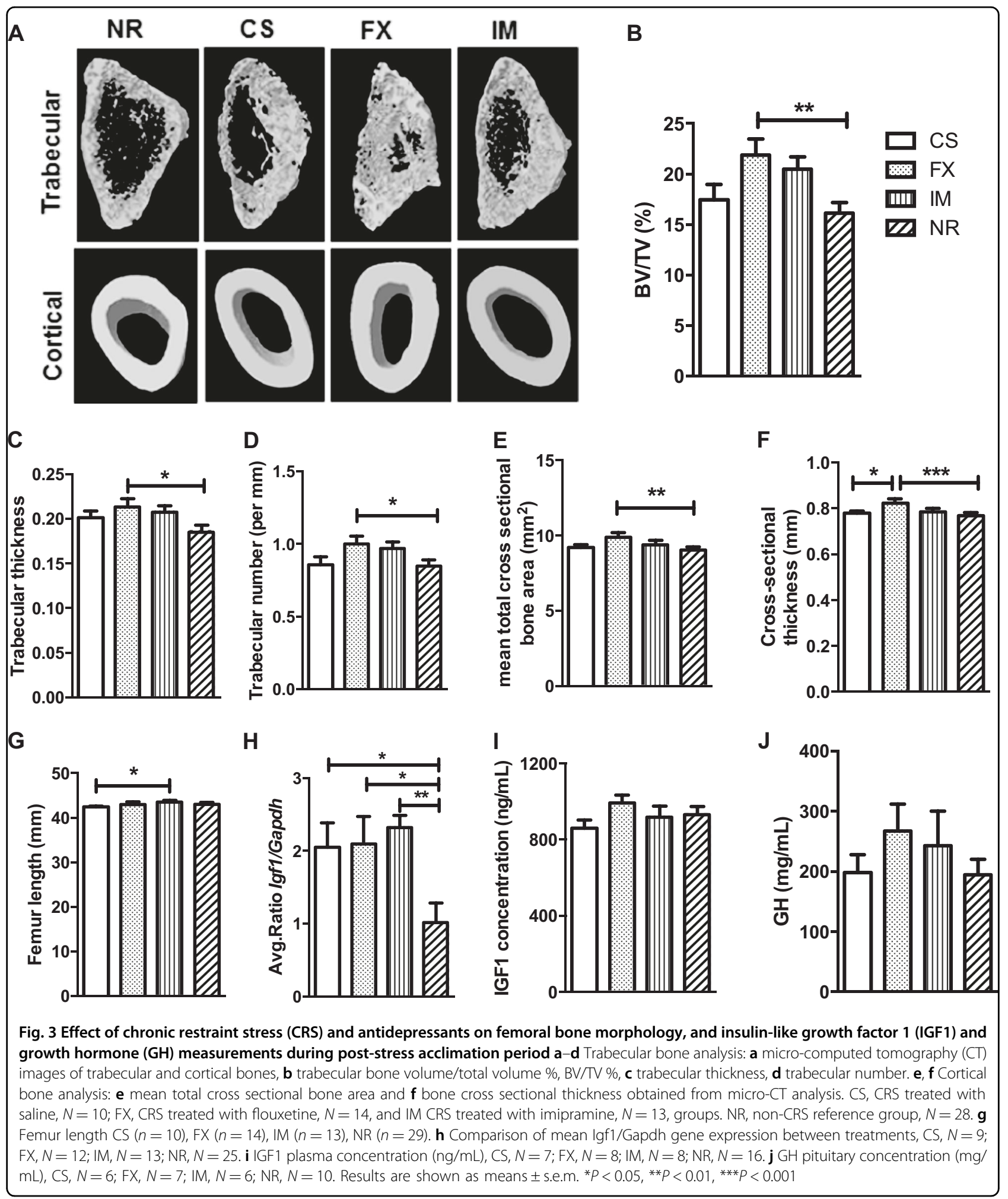




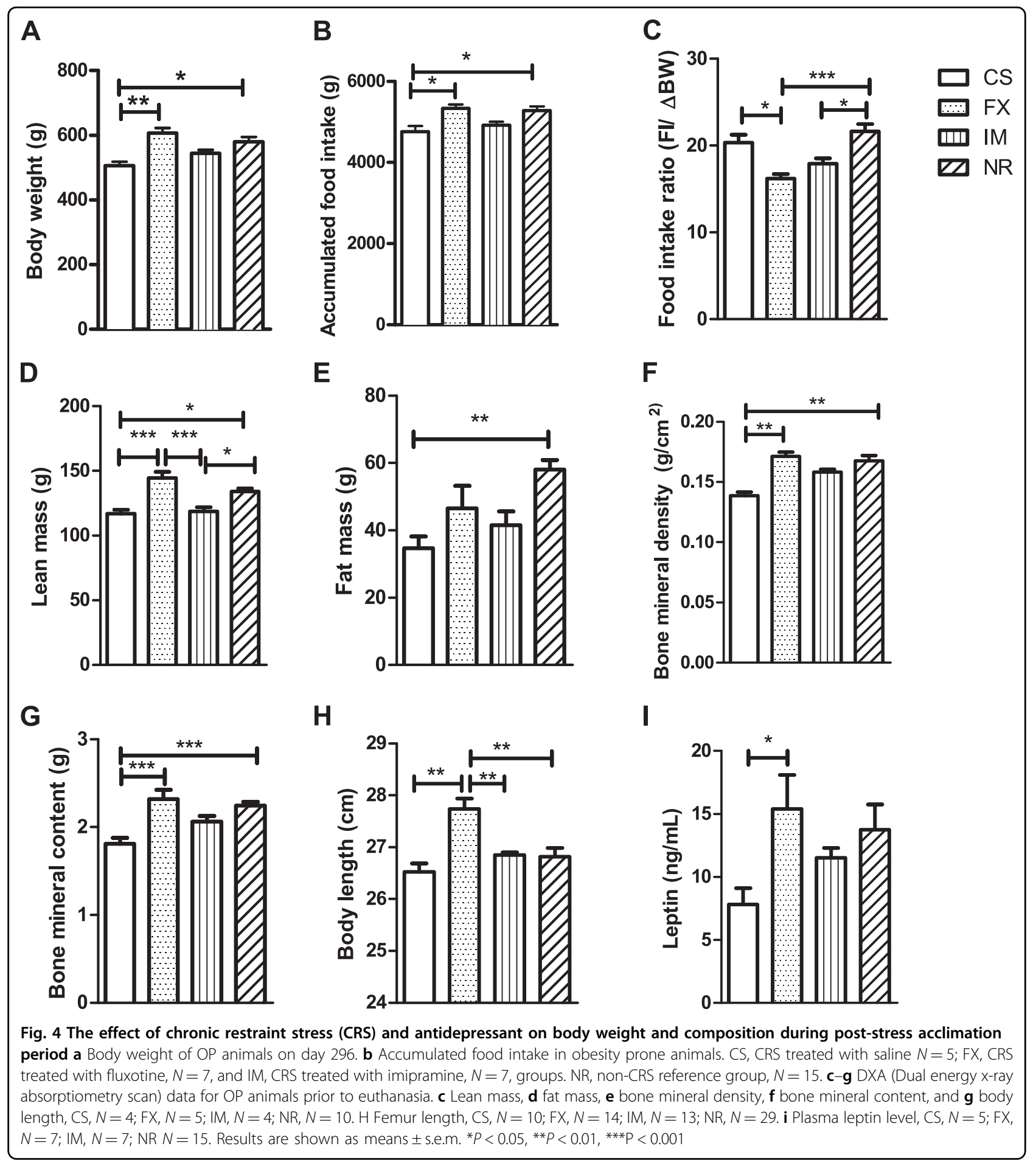

Chronic stressed obesity prone saline-treated animals had worse bone features, and obesity prone FX-treated animals had enhanced bone features and axial length

Stressed animals treated with saline (obesity-prone CS) had lower BMD and BMC in the DXA scan, in comparison to the obesity-prone NR and obesity-prone FX subgroups (respectively, BMD: $P<0.01, P<0.01$, and BMC: $P$
$<0.001, P<0.001$, Fig. 4f-g, and SI Appendix, Table S2). Obesity-prone FX-treated animals had increased axial length in comparison to the other obesity-prone subgroups $(P<0.01$ for all comparisons, Fig. $4 \mathrm{~h}$, and SI Appendix, Table S2). Within obesity-resistant groups, there was no significant difference in axial length, fat mass, BMD, or BMC (SI Appendix, Table S2). 


\section{Chronic stressed obesity prone FX-treated animals had increased leptin plasma levels and were associated with body composition outcomes}

Within CRS obesity-prone subgroups, plasma leptin level was significantly elevated in the FX obesity-prone subgroup compared to the CS obesity-prone subgroup ( $P$ $<0.05$, Fig. 4i, and SI Appendix, Table S2). Leptin was strongly associated with all five body composition outcomes: BMC, BMD, fat percentage, body length, and body weight (respectively, $P=0.001, P=0.009, P<0.0001, P<$ 0.0001 , and $P<0.0001$, Fig. $5 \mathrm{a}-\mathrm{e}$, and SI Appendix, Table S4). The interaction between leptin and antidepressant treatment was significant for BMC $(P=0.009$, Fig. $5 f-h$, and SI Appendix, Table S4). We also found weak evidence for an interaction between triglycerides and antidepressant treatment in body length $(P=0.05$, SI Appendix, Table S4).

\section{Triglyceride plasma levels were lower in chronic stressed} saline- and FX-treated animals

In all the groups, we measured plasma lipid profile (SI Appendix, Fig. S2), and we found that only triglyceride level was significantly different; CS and FX groups had lower tryglyceride levels in comparison to the NR group (Fig. S3A, $P<0.001$ and $P<0.05$, respectively).

\section{Chronic stressed antidepressant-treated animals had increased lipolysis in white adipose tissue}

The relative expression levels for the 8 target genes were quantified in epididymal fat pad samples using qPCR. No significant differences were observed between groups in the level of expression of any of the genes of interest except for the Lipe gene, for which the IM and FX groups were found to have a higher mean expression level than the NR group (Fig. S4, and SI Appendix, Table S5).

\section{Discussion}

Weight gain is a common outcome of antidepressant treatment in the clinical setting, but several studies have shown that chronic administration of various antidepressants results in failure to gain weight or "paradoxical" weight loss in rats, especially at high doses ${ }^{20,21}$. Until recently, the lack of an appropriate animal paradigm caused gaps in the understanding of the pathways involved in antidepressant-induced weight gain in clinical populations. We have developed the SADIO model to help expand our understanding of the interface between obesity, MDD, and antidepressants. This model has allowed the understanding of the long-term impact of the combined effects of limited exposure to recurrent stress and antidepressant ( 2 weeks), followed by chronic intake of high-fat diet ( $>9$ months) on body composition and bone morphology. In this study, we evaluated body weight recovery patterns, body composition, bone features, biochemical and gene expression measurements during the post-stress acclimation period, which is a recovery phase in which the animal is free from the stressor exposure $^{17}$. In agreement with previous literature, 2 weeks of CRS induced significant weight loss in our animals (SI Appendix, Fig. S2) ${ }^{22}$. Within the studied timeframe, the control (CS) group did not attain full body weight recovery in comparison to the NR group, while both antidepressant-treated groups had much better body weight recovery. The ability of the SADIO model to recover from CRS-induced weight loss during the poststress acclimation period could be referred to as a phenomenon of "catch-up growth", which is growth that occurs after a period of growth-inhibiting conditions ${ }^{23}$. Our results suggest that CRS and antidepressant treatment resulted in a period of greater rate of catch-up growth during the post-stress acclimation period. The IM group made full body weight recovery and the FX group had an exacerbated body weight recovery, recovery weights of the FX groups were significantly larger than that of the CS group. These findings suggest that antidepressant-treated groups had better compensatory responses that are associated with induction of hyperphagia, elevated body weight, and repletion of energy reserves leading to super abnormal linear growth ${ }^{23}$.

We found that the obesity-prone FX animals had increased caloric intake, were lengthier and heavier, and had increased leptin levels, yet had significantly lower food intake ratio, suggesting that they had greater ability to store energy. The exacerbated weight gain in the FX group was associated with significant increase in lean mass, BMD, and BMC, and pronounced positive changes in bone cortical and trabecular morphology. Congruently, 9 months (endpoint) after stress and antidepressant use were ceased in the SADIO model, the obesity-prone FX subgroup had significantly greater body length in comparison to the obesity-prone NR and obesity-prone CS subgroups, implying that the propensity to gain weight can influence the rate of catch-up growth and bone allometry. On the other hand, although plasma leptin level was elevated in the obesity-prone FX subgroup in comparison to the obesity-prone CS subgroup, the obesity-prone FX subgroup did not have significant increase in fat mass; yet, the fat mass was significantly lower in the obesity-prone CS subgroup in comparison to the obesity-prone NR subgroup. Furthermore, the metabolic and lipid plasma profiles were not significantly elevated in the FX group, suggesting that there was no further metabolic/lipid dysregulation associated with FXinduced weight gain (SI Appendix, Fig. S3). Our gene expression data suggest that it is unlikely that adipose tissue inflammation and metabolism were meaningfully altered by antidepressant treatment in the SADIO paradigm, as we found no group differences in the expression 
A

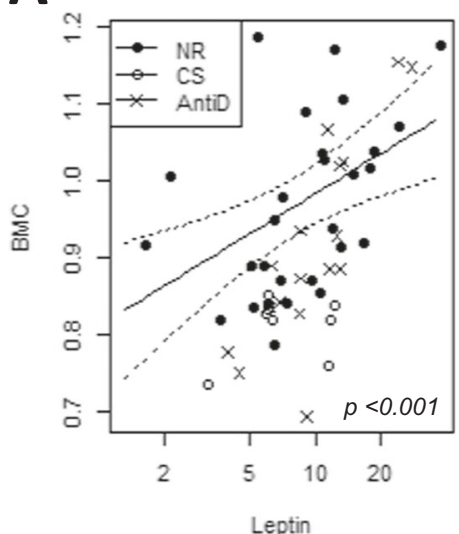

D

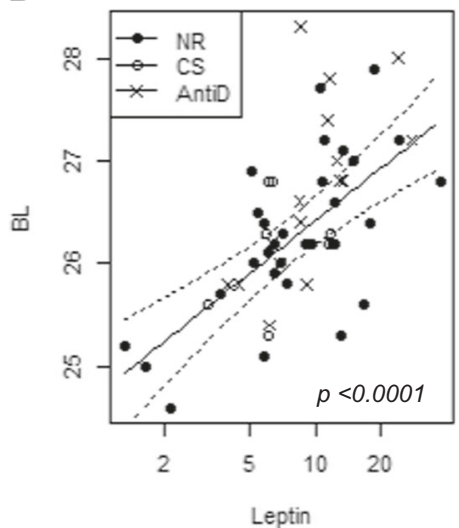

F

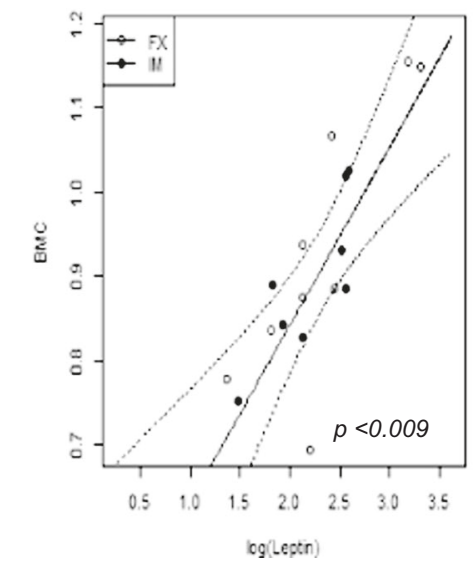

B

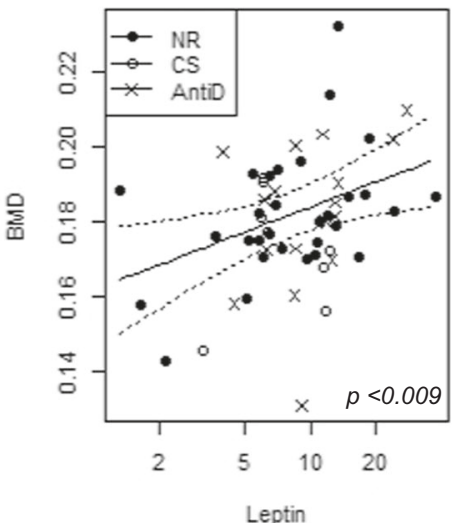

E

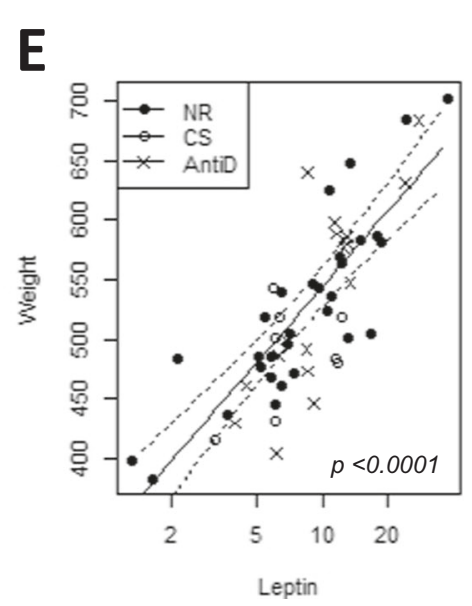

G

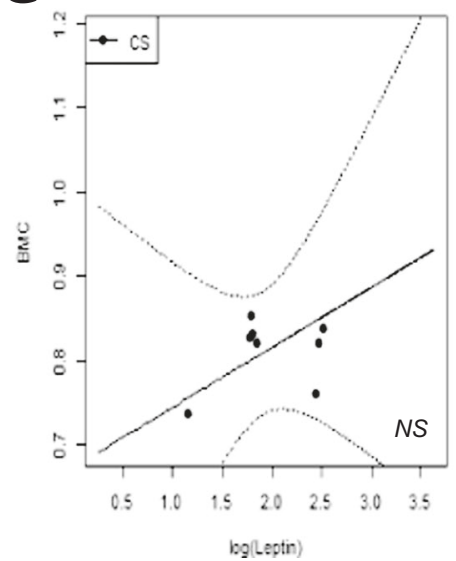

C

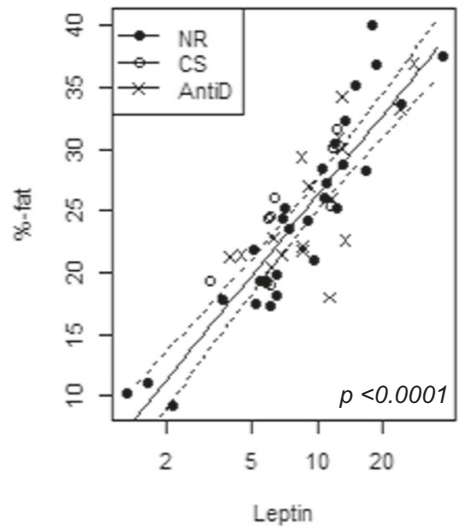

H

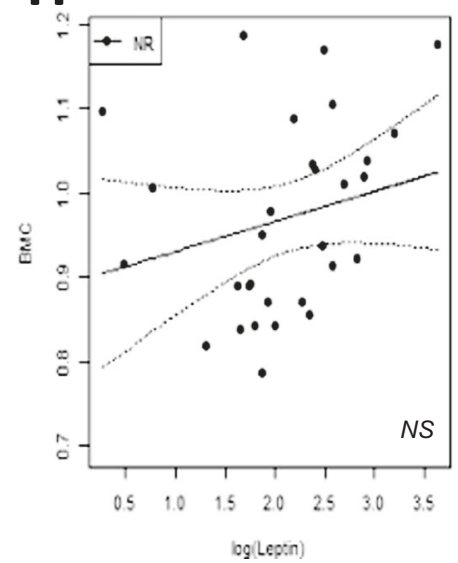

Fig. 5 Associations between leptin and body composition outcomes a BMC, bone mineral content; $\mathbf{b}$ BMD, bone mineral density; c fat percentage; $\mathbf{d} \mathrm{BL}$, body length; e body weight. $\mathrm{NR}=$ non-restraint control group, $N=30 ; \mathrm{CS}=$ chronic restraint stress (CRS) group treated with saline, $N=8 ;$ AntiD $=$ CRS + antidepressant-treated group, $N=17$. $\mathbf{f}-\mathbf{h}$ The interaction between leptin and BMC with its treatment outcomes; $\mathbf{f}$ leptin levels were strongly associated with BMC in the fluoxetine- (FX) and imipramine- (IM) treated animals; leptin levels were not signifincantly associated in the $\mathbf{g}$ CS group and in the $\mathbf{h}$ NR group. AntiD = includes FX and IM 
of genes that indicate adipose tissue inflammation (Tnf) and insulin sensitivity (Slc2a4, Adipoq and Pparg). Moreover, the expression of a gene involved lipolysis (Lipe) was significantly elevated in the FX and IM groups, indicating that there may have been enhanced lipolysis activity in these groups.

The literature on the effect of long-term fluoxetine use on bone metabolism remains controversial. Ortuno et al. ${ }^{24}$ have shown that long-term FX treatment results in serotonin-dependent rise in sympathetic output that increases bone resorption sufficiently to counteract the local anti-resorptive effect. Our result shows that FX treatment and stress for 2 weeks in the context of longterm high-fat diet (over 276 days) resulted in increased body weight and length, accompanied by enhanced femoral bone features. Thus, the mechanism of SSRI action on bone after its discontinuation, in the presence of high-fat diet, is likely to be different from that of SSRI treatment alone, as our findings of elevated BV/TV and trabecular number are compatible with both reduced bone resorption, intensified bone formation (greater trabecular thickness) and increased cortical bone features and bone/body length suggesting enhanced bone growth. Regardless of treatment duration, antidepressant treatment most likely played a role during catch-up growth in the post-stress acclimation period. Furthermore, there was a strong correlation between plasma leptin level and $\mathrm{BMC}$ in the antidepressant-treated groups, which was absent in the NR or the CS control groups. Both, peripheral and central effects of leptin have been described to play a role in bone formation ${ }^{25,26}$. Peripheral leptin has been reported to have direct bone anabolic function and increase osteoblast number and activity ${ }^{27}$; however, via an indirect hypothalamic-mediated pathway, centrally leptin inhibits bone formation by regulating both phases of bone remodeling, resorption and, formation ${ }^{28-30}$, which suggest that short-term antidepressant treatment either lessened the central inhibitory and/or enhanced the peripheral role of leptin in bone formation in the SADIO model, resulting in enhanced bone features.

Weight gain can be induced by mechanisms other than increased fat mass, and growth induced via the GH/IGF1; somatotropic axis is an important factor in weight gain. Wu et al. (2009) demonstrated that the over-expression of systemic IGF1 resulted in enhanced morphological bone features that resemble those of the SADIO model, such as increased cortical thickness and total bone mineral density, and enhanced trabecular bone volume ${ }^{31,32}$. However, we found no significant differences in the endpoint IGF-1 plasma level between the groups; nevertheless, the secretion of GH declines with age and GH/IGF-1 levels may have been different in the earlier stage of weight gain $^{33,34}$.
In this study, we show that IGF1 mRNA levels in the liver are elevated in the CS group than in comparison to the NR group. Although there was no significant difference in systemic IGF-1 level between the groups, significantly lower IGF1 mRNA level in liver in NR group could provide explanation that NR group did not have enhanced bone morphological features compared to CS group. Sjögren et al. (2002) showed that inactivation of liver IGF1 induced a decrease in cortical cross-sectional area and periosteal circumference in the mid-diaphyseal region of the femur and was associated with weaker bone in the mechanical loading test ${ }^{35}$. Thus, weight gain of NR group could be due to diet-induced obesity rather than the growth associated with enhanced bone morphological features.

Previous findings have suggested that short-term FX treatment induces weight loss ${ }^{36}$. While on the one hand, serotonin receptors $5-\mathrm{HT}_{2 \mathrm{~A}}$ and $5-\mathrm{HT}_{2 \mathrm{c}}$ have been proposed to play a significant role in the inhibition of appetite and food intake ${ }^{37}$, on the other hand, long-term FX treatment has been shown to lead to weight gain ${ }^{10-12,36,38}$. Despite numerous studies, the effects of FX treatment in weight regulation remain elusive. Adherence to antidepressant treatment is low; in a large European study of 7525 patients, 56\% abandoned treatment within 4 months, and weight gain was a major side-effect of antidepressant. Antidepressants including mirtazapine ${ }^{39}$, paroxetine $^{40}$, and amitriptyline ${ }^{41}$ have been associated with long-term weight gain, and meta-analysis showed that amitriptyline and paroxetine induced the greatest weight gain in periods over 4 months; $^{36}$ thus, future investigations could address the effects of these antidepressant drugs on weight regulation in the SADIO model.

There are several limitations and future directions for this study. Although our study indicated that stress and antidepressant (SAD)-induced weight gain is associated with growth, we could not clearly show whether the somatotropic axis is involved. We only measured GH/ IGF-1 levels at the end of the experiment and as animals got older, the growth rate was sustained at much slower rate than in earlier periods. In the future, pulsatile $\mathrm{GH}$ levels in plasma should be characterised during an earlier period when a higher growth rate is observed, in order to obtain a better understanding of the mechanism behind SAD-related weight gain.

In the SADIO model, $6 \mathrm{~h}$ of CRS unavoidably may have resulted in food deprivation. Although restraint stress was applied during the light cycle, the effects of food restriction experience on diet-induced weight gain and bone formation cannot be ruled out. For future studies, one additional group with $6 \mathrm{~h}$ of food deprivation should be included in the experimental design. 
Our findings of decreased bone structural features after stress are compatible with those described for major depression and chronic stress-induced depressive-like behavior leading to bone loss ${ }^{42-45}$. Furthermore, Yirmiya $\mathrm{R}$ et al. (2006) have shown that chronic stress decreased osteoblast numbers and bone formation via activation of the sympathetic nervous system, and co-treatment with IM attenuated bone loss only in animals that improved from depressive-like behaviors (responders) ${ }^{45}$. However, to our knowledge this is the first report on the longlasting effects of short-antidepressant treatment in body growth and bone features, which may manifest dissimilarly in subsets of the population with different propensity to gain weight while consuming high-fat diets. As osteoporosis is a condition with significant morbidity and increasing prevalence due to the aging population ${ }^{46,47}$, our findings demonstrating that environmental factors may modulate the role of leptin, may contribute to the understanding of aspects relevant to the heterogeneity and treatement approaches of this condition ${ }^{48}$.

This study has several key findings: (1) Weight gain after recurrent stress and short-term antidepressant treatment followed by diet-induced obesity is different from that of the classic diet-induced obesity. (2) Short-term antidepressant treatment has long-term consequences, and ameliorated the effects of chronic stress on body growth (FX), had long lasting effects in anxiety-like behavior (FX) and adipose tissue gene expression (FX and IM), and affected bone allometric processes that lead to exacerbated bone growth (FX/axial and IM/femur) and enhanced bone structural features (FX). (3) Those effects on body growth and bone features are highly associated with leptin levels. (4) There is a significant interaction between short-term antidepressant treatment and leptin, that likely lessened the central role of leptin in inhibiting bone formation; thus, pharmacological agents may influence the role of leptin in long-term bone regulation. Clinical studies monitoring body weight, caloric intake, and body and bone features after antidepressant discontinuation are needed to confirm our observations in the clinical setting.

\section{Acknowledgements}

We thank Andrea Stojakovic and Shuyu Guo for assisting with animal experiments. This work was supported by grant APP1070935 (to MLW and SRB) from the National Health and Medical Research Council (Australia) and institutional funds from the Australian National University, the South Australian Health and Medical Research Institute, and Flinders University.

\section{Author details}

'John Curtin School of Medical Research, College of Health and Medicine, Australian National University, Canberra, ACT 0200, Australia. ${ }^{2}$ Neuroscience Group (NeUROS), Institute of Translational Medicine, School of Medicine and Health Sciences, Universidad del Rosario, Bogotá, Colombia. ${ }^{3}$ Trauma and Orthopaedic Research Laboratory, Department of Surgery, Medical School, Australian National University, Canberra, ACT 0200, Australia. ${ }^{4}$ Mind \& Brain
Theme, South Australian Health and Medical Research, Adelaide, PO Box 11060 , Adelaide, SA 5001, Australia. ${ }^{5}$ School of Medicine, University of Adelaide, Adelaide, SA 5005, Australia. ${ }^{6}$ Flinders University College of Medicine and Public Health, Bedford Park, SA 5042, Australia. ${ }^{7}$ Freemasons Foundation Centre for Men's Health, Department of Medicine, School of Medicine, University of Adelaide, Adelaide, SA 5005, Australia. ${ }^{8}$ Clinical Orthopaedic Surgery, The Canberra Hospital, Yamba Drive, Garran, ACT 2605, Australia. ${ }^{9}$ Medical Clinic III, Carl Gustav Carus University Hospital, Dresden University of Technology, Fetscherstraße 74, 01307 Dresden, Germany. ${ }^{10}$ Present address: Section on Neural Gene Expression, National Institute of Mental Health, Building 49, Room 5A51, 49 Convent Drive, Bethesda, MD 20892, USA.

${ }^{11}$ Present address: Janssen Australia, 1-5 Khartoum Rd, North Ryde, NSW 2113, Australia. ${ }^{12}$ Present address: State of New York University, Upstate Medical University, Office of the Dean of Medicine, Room 1256 Weiskottem Hall, 766 Irving Ave, Syracuse, NY 13210, USA. ${ }^{13}$ Present address: State of New York, Upstate Medical University, 3738C NRB 505 Irving Ave, Syracuse, NY 13210, USA

\section{Conflict of interest}

G.P.F. is currently employed by Janssen Australia and New Zealand (JanssenCilag Pty Ltd), which is in the business of commercializing therapeutics for depression.

\section{Publisher's note}

Springer Nature remains neutral with regard to jurisdictional claims in published maps and institutional affiliations.

Supplementary Information accompanies this paper at (https://doi.org/ 10.1038/s41398-018-0351-z).

Received: 2 April 2018 Revised: 1 August 2018 Accepted: 9 December 2018 Published online: 16 January 2019

\section{References}

1. Kupfer, D. J., Frank, E. \& Phillips, M. L. Major depressive disorder: new clinical, neurobiological, and treatment perspectives. Lancet 379, 1045-1055 (2012).

2. American Medical Association (AMA). Recognition of obesity as a disease. Contract No.: AMA Health Policy H-440.842 (2013). https://policysearch.amaassn.org/policyfinder/detail/H-440.842?utrl=\%2FAMADoc\%2FHOD.xml-0-3858.xml.

3. WHO. World Health Organization depression fact sheet number 369 (2015). https://www.who.int/en/news-room/fact-sheets/detail/depression.

4. NCHS. Health United States, 2016: With Chartbook on Long-term Trends in Health (National Center for Health Statistics, Centers for Disease Control and Prevention, U.S. D.H.H.S., Hyattsville, 2017)

5. Stunkard, A. J., Fernstrom, M. H., Price, A., Frank, E. \& Kupfer, D. J. Direction of weight change in recurrent depression. Consistency across episodes. Arch. Gen. Psychiatry 47, 857-860 (1990).

6. Markowitz, S., Friedman, M. A. \& Arent, S. M. Understanding the relation between obesity and depression: causal mechanisms and implications for treatment. Clin. Psychol. 15, 1-20 (2008).

7. Onyike, C. U., Crum, R. M., Lee, H. B., Lyketsos, C. G. \& Eaton, W. W. Is obesity associated with major depression? Results from the Third National Health and Nutrition Examination Survey. Am. J. Epidemiol. 158, 1139-1147 (2003).

8. Richardson, L. P. et al. A longitudinal evaluation of adolescent depression and adult obesity. Arch. Pediatr. Adolesc. Med. 157, 739-745 (2003).

9. Bjorntorp, P. Do stress reactions cause abdominal obesity and comorbidities? Obes. Rev. 2, 73-86 (2001).

10. Dannon, P. N. et al. A naturalistic long-term comparison study of selective serotonin reuptake inhibitors in the treatment of panic disorder. Clin. Neuropharmacol. 30, 326-334 (2007).

11. Sussman, N., Ginsberg, D. L. \& Bikoff, J. Effects of nefazodone on body weight: a pooled analysis of selective serotonin reuptake inhibitor- and imipraminecontrolled trials. J. Clin. Psychiatry 62, 256-260 (2001).

12. Michelson, D. et al. Changes in weight during a 1-year trial of fluoxetine. Am. J. Psychiatry 156, 1170-1176 (1999).

13. Ferguson, J. M. SSRI antidepressant medications: adverse effects and tolerability. Prim. Care Companion J. Clin. Psychiatry 3, 22-27 (2001). 
14. Goldstein, B. J. \& Goodnick, P. J. Selective serotonin reuptake inhibitors in the treatment of affective disorders--III. Tolerability, safety and pharmacoeconomics. J. Psychopharmacol. 12, S55-S87 (1998).

15. Mackay, F. J. et al. A comparison of fluvoxamine, fluoxetine, sertraline and paroxetine examined by observational cohort studies. Pharmacoepidemiol Drug Saf. 6, 235-246 (1997).

16. Mastronardi, C. et al. Long-term body weight outcomes of antidepressantenvironment interactions. Mol. Psychiatry 16, 265-272 (2011).

17. Subhash Peter, M. C. Understanding the adaptive response in vertebrates: the phenomenon of ease and ease response during post-stress acclimation. Gen. Comp. Endocrinol. 181, 59-64 (2013).

18. Pagliassotti, M. J., Knobel, S. M., Shahrokhi, K. A., Manzo, A. M. \& Hill, J. O. Time course of adaptation to a high-fat diet in obesity-resistant and obesity-prone rats. Am. J. Physiol. 267, R659-R664 (1994).

19. Team, R. C. R. A language and environment for statistical computing. R Foundation for Statistical Computing, Vienna (2017).

20. McAllister, E. J. et al. Ten putative contributors to the obesity epidemic. Crit. Rev. Food Sci. Nutr. 49, 868-913 (2009).

21. File, S. E. \& Tucker, J. C. Behavioral consequences of antidepressant treatment in rodents. Neurosci. Biobehav Rev. 10, 123-134 (1986).

22. Watanabe, Y., Gould, E. \& McEwen, B. S. Stress induces atrophy of apical dendrites of hippocampal CA3 pyramidal neurons. Brain Res. 588, 341-345 (1992).

23. Baron, J. et al. Catch-up growth after glucocorticoid excess: a mechanism intrinsic to the growth plate. Endocrinology 135, 1367-1371 (1994).

24. Ortuno, M. J. et al. Serotonin-reuptake inhibitors act centrally to cause bone loss in mice by counteracting a local anti-resorptive effect. Nat. Med. 22, 1170-1179 (2016)

25. Luther, J. \& David, J. P. Bone and adipose tissue formation. Z. Rheumatol. 75, 701-706 (2016).

26. Whitfield, J. F. Leptin: brains and bones. Expert Opin. Investig. Drugs 10, 1617-1622 (2001).

27. Cornish, J. et al. Leptin directly regulates bone cell function in vitro and reduces bone fragility in vivo. J. Endocrinol. 175, 405-415 (2002).

28. Ducy, P. et al. Leptin inhibits bone formation through a hypothalamic relay: a central control of bone mass. Cell 100, 197-207 (2000).

29. Shi, Y. et al. Dissociation of the neuronal regulation of bone mass and energy metabolism by leptin in vivo. Proc. Natl. Acad. Sci. Usa. 105, 20529-20533 (2008).

30. Yadav, V. K. et al. A serotonin-dependent mechanism explains the leptin regulation of bone mass, appetite, and energy expenditure. Cell 138, 976-989 (2009).

31. Wu, Y., Sun, H., Yakar, S. \& LeRoith, D. Elevated levels of insulin-like growth factor (IGF)-I in serum rescue the severe growth retardation of IGF-I null mice. Endocrinology 150, 4395-4403 (2009).
32. Elis, S. et al. Elevated serum levels of IGF-1 are sufficient to establish normal body size and skeletal properties even in the absence of tissue IGF-1. J. Bone Miner. Res. 25, 1257-1266 (2010).

33. Corpas, E., Harman, S. M. \& Blackman, M. R. Human growth hormone and human aging. Endocr. Rev. 14, 20-39 (1993).

34. Iranmanesh, A., Lizarralde, G. \& Veldhuis, J. D. Age and relative adiposity are specific negative determinants of the frequency and amplitude of growth hormone $(\mathrm{GH})$ secretory bursts and the half-life of endogenous $\mathrm{GH}$ in healthy men. J. Clin. Endocrinol. Metab. 73, 1081-1088 (1991).

35. Sjögren, K. et al. Effects of liver-derived Insulin-like Growth Factor-I on bone metabolism in mice. J. Bone Mineral. Res. 17, 1977-1987 (2002).

36. Serretti, A. \& Mandelli, L. Antidepressants and body weight: a comprehensive review and meta-analysis. J. Clin. Psychiatry 71, 1259-1272 (2010).

37. Malhi, G. S., Mitchell, P. B. \& Caterson, I. 'Why getting fat, Doc?' Weight gain and psychotropic medications. Aust. N. Z. J. Psychiatry 35, 315-321 (2001).

38. Andersen, S. W., Clemow, D. B. \& Corya, S. A. Long-term weight gain in patients treated with open-label olanzapine in combination with fluoxetine for major depressive disorder. J. Clin. Psychiatry 66, 1468-1476 (2005).

39. Montgomery, S. A., Reimitz, P. E. \& Zivkov, M. Mirtazapine versus amitriptyline in the long-term treatment of depression: a double-blind placebo-controlled study. Int Clin. Psychopharmacol. 13, 63-73 (1998).

40. Dannon, P. N. et al. Three year naturalistic outcome study of panic disorder patients treated with paroxetine. BMC Psychiatry 4, 16 (2004).

41. Coppen, A. et al. Continuation therapy with amitriptyline in depression. Br. J. Psychiatry 133, 28-33 (1978).

42. Michelson, D. et al. Bone mineral density in women with depression. N. Engl. J. Med. 335, 1176-1181 (1996).

43. Yazici, K. M., Akinci, A., Sutcu, A. \& Ozcakar, L. Bone mineral density in premenopausal women with major depressive disorder. Psychiatry Res. 117 271-275 (2003).

44. Kahl, K. G. et al. Bone mineral density, markers of bone turnover, and cytokines in young women with borderline personality disorder with and without comorbid major depressive disorder. Am. J. Psychiatry 162, 168-174 (2005).

45. Yirmiya, R. et al. Depression induces bone loss through stimulation of the sympathetic nervous system. Proc. Natl. Acad. Sci. Usa. 103, 16876-16881 (2006).

46. Amin, S., Achenbach, S. J., Atkinson, E. J., Khosla, S. \& Melton, L. J. III Trends in fracture incidence: a population-based study over 20 years. J. Bone Miner. Res. 29, 581-589 (2014)

47. Cooper, C., Campion, G. \& Melton, L. J. III Hip fractures in the elderly: a worldwide projection. Osteoporos. Int. 2, 285-289 (1992).

48. Brommage, R. et al. Adult Tph2 knockout mice without brain serotonin have moderately elevated spine trabecular bone but moderately low cortical bone thickness. Bone. Rep. 4, 718 (2015). 